\title{
Enhanced sensitivity of low-frequency signal by using broad squeezed light and bichromatic local oscillator
}

\author{
Wei Li, ${ }^{1,2}$ Yuanbin Jin,,${ }^{1,2}$ Xudong Yu, ${ }^{1,2}$ and Jing Zhang, ${ }^{\dagger 1,3}$ \\ ${ }^{1}$ State Key Laboratory of Quantum Optics and Quantum Optics Devices, \\ Institute of Opto-Electronics, Shanxi University, Taiyuan 030006, P.R.China \\ ${ }^{2}$ Collaborative Innovation Center of Extreme Optics, Shanxi University, Taiyuan 030006, P.R.China \\ ${ }^{3}$ Synergetic Innovation Center of Quantum Information and Quantum Physics, \\ University of Science and Technology of China, Hefei, Anhui 230026, P. R. China
}

\begin{abstract}
We experimentally study a protocol of using the broadband high-frequency squeezed vacuum to detect the low-frequency signal. In this scheme, the lower sideband field of the squeezed light carries the low-frequency modulation signal and the two strong coherent light fields are applied as the bichromatic local oscillator in the homodyne detection to measure the quantum entanglement of the upper and lower sideband for the broadband squeezed light. The power of one of local oscillators for detecting the upper sideband can be adjusted to optimize the conditional variance in the low frequency regime by subtracting the photocurrent of the upper sideband field of the squeezed light from that of the lower sideband field. By means of the quantum correlation of the upper and lower sideband for the broadband squeezed light, the low-frequency signal beyond the standard quantum limit is measured. This scheme is appropriate for enhancing sensitivity of low-frequency signal by the aid of the broad squeezed light, such as gravitational waves detection, and does not need to directly produce the low frequency squeezing in optical parametric process.
\end{abstract}

The first detection of gravitational waves (GW) emitted from the merger of two black holes by the Laser Interferometer Gravitational-Wave Observatory (LIGO) sets the course of a new era of astrophysics. GW detection is now opening an exciting new observational frontier in astronomy and cosmology [1 $[3]$. The further improvement of GW detector sensitivity is expected to extend the detection range and the event rate of binary black holes coalescence, and may lead to detections of more exotic sources.

In Advanced LIGO, vacuum fluctuations entering from the dark port of the interferometer [4] can make the quadrature phase of the output carrier field at the dark port noisy, while which contains GW signal. However as the squeezed vacuum state is fed into the dark port of the interferometer, the sensitivity can be improved beyond the standard quantum limit (SQL) [5, 6]. The use of squeezed states to enhance the sensitivity began with initial proof-of-principle experiments and recently have been demonstrated in GEO 600 [7] and LIGO [8]. Since terrestrial GW signal locates in $10 \mathrm{~Hz}$ to $10 \mathrm{kHz}$ band [9, 10], the squeezing in the audio band is required, which is a great technical challenge. Until now, there is a very wide research demonstrating the squeezing at the lower frequency band [11 13] and applications in quantum metrology 14 24].

Broadband squeezing has been demonstrated at megahertz frequencies, where technical noise sources of the laser light are not present. At these frequencies, the laser operates at or near the shot-noise limit. Due to the strong quantum correlation between the lower and upper sideband of the squeezed light field, the single broadband squeezed light can be split into $\mathrm{N}$ pairs of upper and lower sideband fields with spatial separation to produce N independent Einstein-Podolsky-Rosen (EPR) entangled fields [25]. This scheme was demonstrated experimentally by using a pair of frequency-shifted local oscillators to measure this EPR entanglement [26, 27]. Recently, a theoretical protocol is proposed to improve LIGO's sensitivity beyond the SQL via EPR entanglement of the broad squeezing field and the dual use of the interferometer as both the GW detector and the filter, eliminating the need for external narrow filter cavities 28]. In this paper, we employ a broadband high-frequency squeezed vacuum to detect low-frequency signal beyond the standard quantum limit. The broadband squeezed vacuum consists a pair of EPR entangled beams: the signal beam (lower sideband field) around the carrier frequency $\omega_{0}$, and the idler beam (upper sideband field) around $\omega_{0}+\Lambda$. The lower sideband field around the carrier frequency $\omega_{0}$ will carry the low-frequency modulation signal around the carrier frequency $\omega_{0}$, however, the upper sideband field around $\omega_{0}+\Lambda$ feel nothing. The output lower and upper sideband fields may be separated in space by a mode cleaner cavity and measured by homodyne detection with two local oscillators at frequency $\omega_{0}$ and $\omega_{0}+\Lambda$ respectively. The conditional squeezing of the output signal beam can be obtained by subtracting the photocurrent of the idler beam from that of the signal beam. Here, the lower and upper sideband fields of the broad squeezed light may be separated in space by a mode cleaner cavity before or after carrying the low-frequency modulation signal. Thus this scheme also can be considered as: 1) First, the broadband squeezed vacuum is separated into the lower and upper sideband fields in space by a mode cleaner cavity. 2) The lower sideband field around the carrier frequency $\omega_{0}$ is sent into sensitive device (such as the interferometer), therefore, the low-frequency sig- 
nal around the carrier frequency $\omega_{0}$ is added in the lower sideband field by the sensitive device. 3) The lower and upper sideband fields are measured by homodyne detection with two local oscillators at frequency $\omega_{0}$ and $\omega_{0}+\Lambda$ respectively. The conditional variance of the lower sideband beam can be obtained by subtracting the photocurrent of the upper sideband beam from that of the lower sideband beam. Thus this scheme can avoid producing the low frequency squeezing to improve the sensitivity of the interferometer.

In this paper, we remove the mode cleaner cavity to separate the signal and idler beams in space and utilize a bichromatic local oscillator (BLO) to directly detect the signal and idler beams of a broad high-frequency squeezed vacuum after carrying the low-frequency signal by combining with a phase-modulated coherent light at around $\omega_{0}$ on a beam splitter. This scheme can avoid optical losses introduced by the mode cleaner cavity. Moreover by optimally adjusting the power of one of local oscillator for detecting the idler field, we can obtain the minimum conditional variance of the signal beam and improve the signal noise ratio. The theoretical scheme based on BLO to detect the squeezed state was proposed [29] and the phase-sensitive detection with a BLO or a double-sideband signal field were studied experimentally 30 32. And the measurement of a broad squeezed vacuum state by means of a BLO was demonstrated experimentally [33, 34]. Recently, by making use of the multi-frequency homodyne detection, the experiments of cross-frequency entanglements generated in periodically pumped OPOs have been reported [35, 36].

The schematic diagram of the detection is shown in Fig. 1(a). A BLO with two local oscillators at frequency $\omega_{0}$ and $\omega_{0}+\Lambda$ is mixed with the detected light field at a 50/50 beam splitter. The power of one of local oscillator (upper local oscillator) at $\omega_{0}+\Lambda$ can be adjusted with the factor g. The relative phase $\theta$ of the local oscillator and the detected field can be controlled by the reflective mirror mounted on a PZT (piezoelectric transducer). The annihilation operators of the BLO and the detected signal field can be written as $\hat{a}(t)=\hat{a}_{-}(t) \exp \left[-i \omega_{0} t\right]+\hat{a}_{+}(t) \exp \left[-i\left(\omega_{0}+\Lambda\right) t\right]$ and $\hat{b}(t)=\hat{b}_{0}(t) \exp \left(-i \omega_{0} t\right)$, where $\hat{a}_{+(-)}(t)$ and $\hat{b}_{0}(t)$ are the slow varying operators of the fields. The normalized difference of the photocurrents of the two detectors at the $50 / 50$ beam splitter is

$$
\hat{i}(t)=\frac{1}{a}\left[\left\langle\hat{a}^{\dagger}(t)\right\rangle \hat{b}(t) e^{-i \theta}+\langle\hat{a}(t)\rangle \hat{b}^{\dagger}(t) e^{i \theta}\right],
$$

where the fields satisfy $\left\langle\hat{a}_{+}\right\rangle=g a$, and $\left\langle\hat{a}_{-}\right\rangle=a \gg$ $\left\langle\hat{b}_{0}\right\rangle \sim 0$. Therefore the BLO is a pair of the strong coherent states. And the detected field is the vacuum state or the squeezed vacuum state carrying the low-frequency signal around frequency $\omega_{0}$.

The difference of the photocurrents analyzed at the radio frequency $\Omega$ is expressed as

$$
\hat{i}(\Omega)=\hat{Q}_{-}(\Omega, \theta)+g \hat{Q}_{+}(\Omega, \theta) .
$$

Here, we express the quadrature component of the signal field around the central frequency $\omega_{0}$, which easily compare with the measurement with a single local oscillator $(g=0)$ at $\omega_{0}$. Therefore, the quadrature component of the detected field can be defined as $\hat{Q}_{-}(\Omega, \theta)=\hat{b}\left(\omega_{0}-\right.$ $\Omega) e^{-i \theta}+\hat{b}^{\dagger}\left(\omega_{0}+\Omega\right) e^{i \theta}$, and $\hat{Q}_{+}(\Omega, \theta)=\hat{b}\left(\omega_{0}+\Lambda-\Omega\right) e^{-i \theta}+$ $\hat{b}^{\dagger}\left(\omega_{0}+\Lambda+\Omega\right) e^{i \theta}$. The quadrature amplitude $(\theta=0)$ can be $\hat{X}_{-}(\Omega)=\hat{b}\left(\omega_{0}-\Omega\right)+\hat{b}^{\dagger}\left(\omega_{0}+\Omega\right)$ and the quadrature phase $(\theta=\pi / 2) \hat{Y}_{-}(\Omega)=-i\left[\hat{b}\left(\omega_{0}-\Omega\right)-\hat{b}^{\dagger}\left(\omega_{0}+\Omega\right)\right]$. The arbitrary quadrature component of the detected field can be measured by scanning the relative phase of $\theta$. So when $\theta=0$, the difference of the photocurrents will give the information of the quadrature amplitude of the detected field $\hat{X}_{B}(\Omega)=\hat{X}_{-}(\Omega)+g \hat{X}_{+}(\Omega)$, and when $\theta=\pi / 2$, the quadrature phase $\hat{Y}_{B}(\Omega)=\hat{Y}_{-}(\Omega)+g \hat{Y}_{+}(\Omega)$.

Since a single broadband squeezed light can be split into a pair of upper and lower sideband fields as EPR entangled fields, the minimum conditional variance of the output lower sideband (signal) beam can be obtained with the help of the upper sideband (idler) beam. Considering the simple optical parametric oscillator (OPO) process, the nonlinear medium is pumped with the second-harmonic wave of $\omega_{p}=2 \omega_{0}+\Lambda$. The annihilation operators of the output lower and upper sideband fields of OPO can be written as

$$
\begin{aligned}
& \hat{b}_{-}^{s}=\hat{b}_{-}^{0} \cosh r+\hat{b}_{+}^{\dagger 0} e^{i \theta_{p}} \sinh r, \\
& \hat{b}_{+}^{s}=\hat{b}_{+}^{0} \cosh r+\hat{b}_{-}^{\dagger 0} e^{i \theta_{p}} \sinh r,
\end{aligned}
$$

where $r$ is squeezing factor, $\theta_{p}$ is the phase of the pump field, $\hat{b}_{-}^{0}$ and $\hat{b}_{+}^{0}$ are the annihilation operators of the input lower and upper sideband vacuum fields of OPO with $\left\langle\delta^{2} X_{-}^{0}(\Omega)\right\rangle=\left\langle\delta^{2} X_{+}^{0}(\Omega)\right\rangle=\left\langle\delta^{2} Y_{-}^{0}(\Omega)\right\rangle=\left\langle\delta^{2} Y_{+}^{0}(\Omega)\right\rangle=1$. If $\theta_{p}=0$, the phase amplitudes of the output lower and upper sideband fields of OPO can be given by

$$
\begin{aligned}
\hat{X}_{-}^{s}(\Omega) & =\hat{X}_{-}^{0}(\Omega) \cosh r+\hat{X}_{+}^{0}(\Omega) \sinh r \\
\hat{Y}_{-}^{s}(\Omega) & =\hat{Y}_{-}^{0}(\Omega) \cosh r-\hat{Y}_{+}^{0}(\Omega) \sinh r \\
\hat{X}_{+}^{s}(\Omega) & =\hat{X}_{+}^{0}(\Omega) \cosh r+\hat{X}_{-}^{0}(\Omega) \sinh r \\
\hat{Y}_{+}^{s}(\Omega) & =\hat{Y}_{+}^{0}(\Omega) \cosh r-\hat{Y}_{-}^{0}(\Omega) \sinh r
\end{aligned}
$$

then, the difference and sum of amplitude phase quadratures of the output lower and upper sideband fields of OPO are obtained

$$
\begin{aligned}
\hat{X}_{-}^{s}(\Omega)-\hat{X}_{+}^{s}(\Omega) & =\left(\hat{X}_{-}^{0}(\Omega)-\hat{X}_{+}^{0}(\Omega)\right) e^{-r} \\
\hat{Y}_{-}^{s}(\Omega)+\hat{Y}_{+}^{s}(\Omega) & =\left(\hat{Y}_{-}^{0}(\Omega)+\hat{Y}_{+}^{0}(\Omega)\right) e^{-r} \\
\hat{X}_{-}^{s}(\Omega)+\hat{X}_{+}^{s}(\Omega) & =\left(\hat{X}_{-}^{0}(\Omega)+\hat{X}_{+}^{0}(\Omega)\right) e^{+r} \\
\hat{Y}_{-}^{s}(\Omega)-\hat{Y}_{+}^{s}(\Omega) & =\left(\hat{Y}_{-}^{0}(\Omega)-\hat{Y}_{+}^{0}(\Omega)\right) e^{+r}
\end{aligned}
$$


The variances of the output lower and upper sideband fields of OPO are expressed by

$$
\begin{aligned}
\left\langle\delta^{2} X_{-}^{s}(\Omega)\right\rangle & =\left\langle\delta^{2} X_{+}^{s}(\Omega)\right\rangle=\left\langle\delta^{2} Y_{-}^{s}(\Omega)\right\rangle=\left\langle\delta^{2} Y_{+}^{s}(\Omega)\right\rangle \\
& =\frac{e^{-2 r}+e^{2 r}}{2}
\end{aligned}
$$

and the correlated variances are given by

$$
\begin{aligned}
& \left\langle\delta^{2}\left(\hat{X}_{-}^{s}(\Omega)-\hat{X}_{+}^{s}\right)(\Omega)\right\rangle=\left\langle\delta^{2}\left(\hat{Y}_{-}^{s}(\Omega)+\hat{Y}_{+}^{s}(\Omega)\right)\right\rangle=2 e^{-2 r} \\
& \left\langle\delta^{2}\left(\hat{X}_{-}^{s}(\Omega)+\hat{X}_{+}^{s}\right)(\Omega)\right\rangle=\left\langle\delta^{2}\left(\hat{Y}_{-}^{s}(\Omega)-\hat{Y}_{+}^{s}(\Omega)\right)\right\rangle=2 e^{+2}(\overline{7})
\end{aligned}
$$

The variance of the conditional quadrature phase $\hat{Y}_{B}(\Omega)$ detected by BLO with the factor $\mathrm{g}$ is expressed by

$$
\begin{aligned}
\left\langle\delta^{2} \hat{Y}_{B}(\Omega)\right\rangle & =\left\langle\delta^{2}\left(\hat{Y}_{-}^{s}+g \hat{Y}_{+}^{s}\right)\right\rangle \\
& =\frac{e^{2 r}}{2}(1-g)^{2}+\frac{e^{-2 r}}{2}(1+g)^{2}
\end{aligned}
$$

In parallel, the conditional quadrature amplitude $\hat{X}_{B}(\Omega)$ is given by

$$
\begin{aligned}
\left\langle\delta^{2} \hat{X}_{B}(\Omega)\right\rangle & =\left\langle\delta^{2}\left(\hat{X}_{-}^{s}+g \hat{X}_{+}^{s}\right)\right\rangle \\
& =\frac{e^{-2 r}}{2}(1-g)^{2}+\frac{e^{2 r}}{2}(1+g)^{2} .
\end{aligned}
$$

When we choose the optimized value of $g_{\text {opt }}=\left(e^{2 r}-\right.$ $\left.e^{-2 r}\right) /\left(e^{2 r}+e^{-2 r}\right)$, the minimum conditional variance of the output lower sideband field is obtained

$$
\left\langle\delta^{2} \hat{Y}_{B}^{o p t}(\Omega)\right\rangle=\frac{2}{e^{2 r}+e^{-2 r}} .
$$

Here, the method of obtaining the minimum conditional variance by the optimized factor is same as the previous works [37, 38]. Thus, for the vacuum field injection, $g=0$ (without the upper local oscillator $\omega_{0}+\Lambda$ ) and $\left\langle\delta^{2} \hat{Y}_{B}(\Omega)\right\rangle=1$. When the broadband squeezed light with $3 \mathrm{~dB}$ is injected, the minimum conditional variance is $-0.97 \mathrm{~dB}$ with $g_{\text {opt }}=0.6$.

The experimental setup and schematic diagram are shown in Fig. 1. A diode-pumped intra-cavity frequencydoubled single-frequency laser provides the fundamental light of $200 \mathrm{~mW}$ at $1064 \mathrm{~nm}$ and the second-harmonic light of $450 \mathrm{~mW}$ at $532 \mathrm{~nm}$ simultaneously. The secondharmonic light with the frequency of $\omega_{p}=2 \omega_{0}+\Lambda$ is used to pump an OPO to generate the broad squeezed vacuum field. The OPO cavity is resonant for both the pump light at $532 \mathrm{~nm}$ and the fundamental light at 1064 nm. The OPO cavity with $38 \mathrm{~mm}$ long contains a type-I PPKTP crystal $(1 \mathrm{~mm} \times 2 \mathrm{~mm} \times 10 \mathrm{~mm})$, the front facet of which is highly reflective for $1064 \mathrm{~nm}$ and has a power transmittance of $5 \%$ for $532 \mathrm{~nm}$, and an outcoupling mirror that is highly reflective at $532 \mathrm{~nm}$ and has an intensity transmittance of $12.5 \%$ at $1064 \mathrm{~nm}$. The bandwidth of the OPO is about $70 \mathrm{MHz}$. The OPO cavity is locked
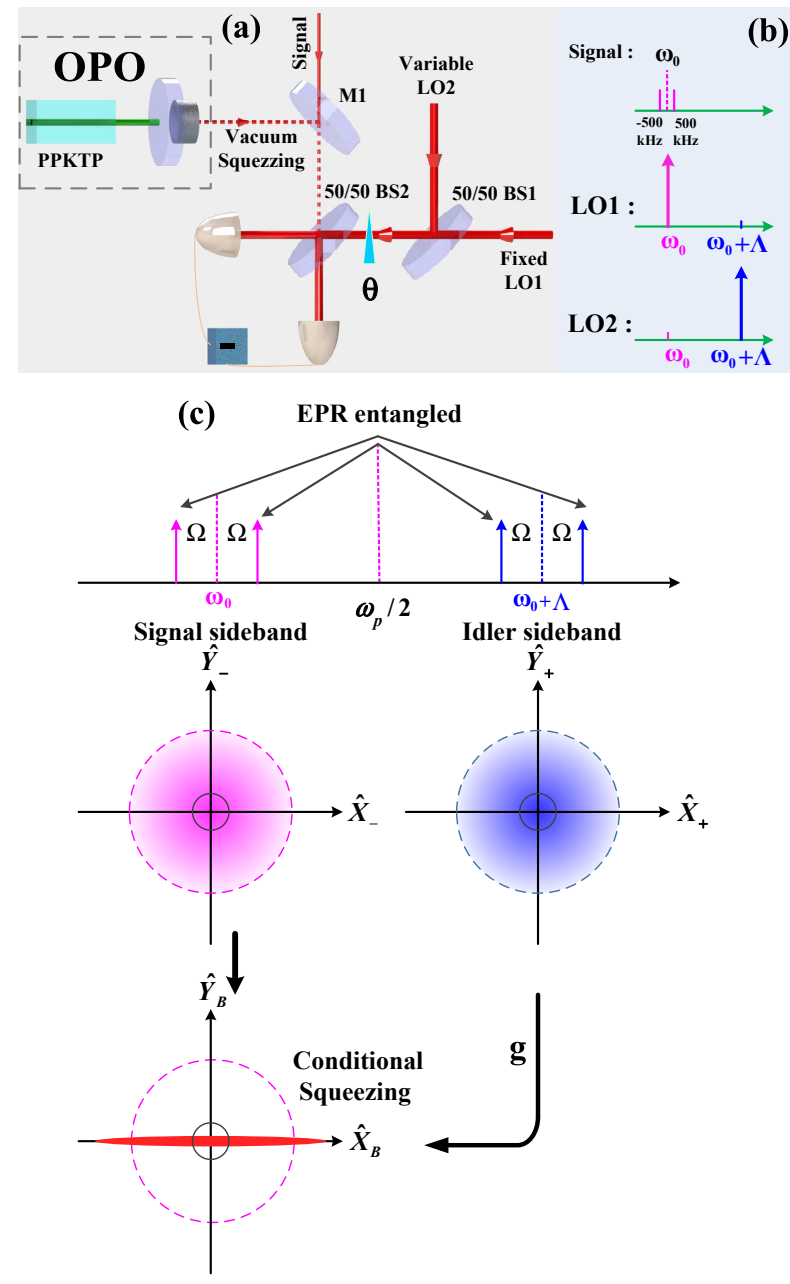

FIG. 1: The experimental setup and schematic diagram of detecting low-frequency signal beyond the standard quantum limit by a broadband squeezing and BLO. a, The experimental setup. OPO, optical parametric oscillator; BS, 50/50 beam splitter; LO, local oscillator; M1, 98/2 beam splitter. b, Spectra of the weak lowfrequency signal and the BLO. c, Spectral decomposition of EPR-entanglement for the broad squeezed light (upper panel) and prepare the conditional squeezing of the signal beam by BLO detection (lower panel).

by PDH (Pound-Driver-Hall) technology and the error signal is extracted by detecting the reflected pump light of the OPO. The output broad squeezed field carries the weak low-frequency signal $( \pm 500 \mathrm{kHz})$ around the carrier frequency $\omega_{0}$ at M1 (98/2 beam splitter).

The fundamental output field $\left(\omega_{p} / 2\right)$ passes through the acousto-optical frequency-shifted system and then is split into two beams with the frequency of $\omega_{0}=$ $\omega_{p} / 2-5 M H z$ and $\omega_{0}+\Lambda=\omega_{p} / 2+5 M H z$ (here, $\Lambda=10 M H z)$. In the frequency-shifted system, AOM1 shifts the laser frequency by the first-order diffraction with a mount of $+110 \mathrm{MHz}$. Then the frequency-shifted laser is split into two parts, which are translated back 
by AOM2 and AOM3 with the mount of $-105 \mathrm{MHz}$ and $-115 \mathrm{MHz}$ respectively. The two frequency-shifted laser beams at $\omega_{p} / 2 \pm 5 \mathrm{MHz}$ as local oscillator (LO) 1 and 2 are combined on $50 / 50 \mathrm{BS}$ with the same polarization to generate the BLO. Here, the power of LO1 is fixed and that of LO2 can be varied. A small portion from the frequency-shifted laser beams at $\omega_{p} / 2-5 \mathrm{MHz}$ is used to generate the weak low-frequency signal by a phase modulator. In order to lock the relative frequency and phase of the two LOs, the signal generators of the acousto-optical frequency-shifted system are locked by the clock synchronization technology [31, 33]. The squeezed light with the weak low-frequency signal is mixed with the BLO on the $50 / 50$ BS. Finally, the two output fields of the BS are detected by two balanced detectors.

Fig. 2 shows the noise variance of the conditional quadrature phase amplitude as the function of the factor $g^{2}$. Here, the noise of the conditional quadrature phase amplitude is normalized to the SQL, which is determined only with the LO1 $(g=0)$ and injecting vacuum field (blocking the squeezed light and signal field). When injecting the squeezed light and given the intensity of the LO2 (given the factor of g), the conditional arbitrary quadrature components are measured by scanning the relative phase of $\theta$. The conditional quadrature phase amplitude as the function of the factor $g^{2}$ (Fig. 2(a)) can be obtained by finding the minimum and maximum noise variance from the measured arbitrary quadrature components, which are good agreement with the theoretical calculation. Fig. 2(a) gives two different squeezing (antisqueezing) with $3.9 \mathrm{~dB}(5.24 \mathrm{~dB})$ and $5.9 \mathrm{~dB}(11.6$ dB) respectively. Here, the extra noise $N_{e}$ of the antisqueezed component can be calculated from the values of squeezing and antisqueezing (see Appendix). Fig. 2(b) and (c) the noise variance of the conditional arbitrary quadrature components with the optimal factor $g_{\text {opt }}$ for two different input squeezing with $3.9 \mathrm{~dB}$ and $5.9 \mathrm{~dB}$ respectively. Thus, the minimum conditional variance is obtained with $-1.5 \mathrm{~dB}$ for the initial squeezing of $3.9 \mathrm{~dB}$ and $-3.1 \mathrm{~dB}$ for that of $5.9 \mathrm{~dB}$. Here, the noise variances of the arbitrary quadrature components of the lower sideband field of the squeezing light $(\mathrm{g}=0)$ are constant and larger than SQL as the function of the relative phase of $\theta$, which demonstrates that one beam of an EPR entangled pair is thermal state.

Fig. 3 shows the enhanced sensitivity of low-frequency signal with $500 \mathrm{kHz}$ around the frequency $\omega_{0}$ by using broad squeezed light and BLO. When the vacuum field is injected, the noise floor (black (gray) curve in Fig. 3 ) of the signal corresponds to SQL with $g=0$. If the squeezed field is injected and $\mathrm{g}=0$, the very noisy floor (blue (light gray) curve in Fig. 3) is the noise variance of one beam of an EPR entangled pair. When we choose the optimal factor $g_{o p t}$, the enhanced sensitivity of lowfrequency signal is obtained and the signal-noise ratio is improved with $1.5 \mathrm{~dB}$ for the initial broad squeezing of

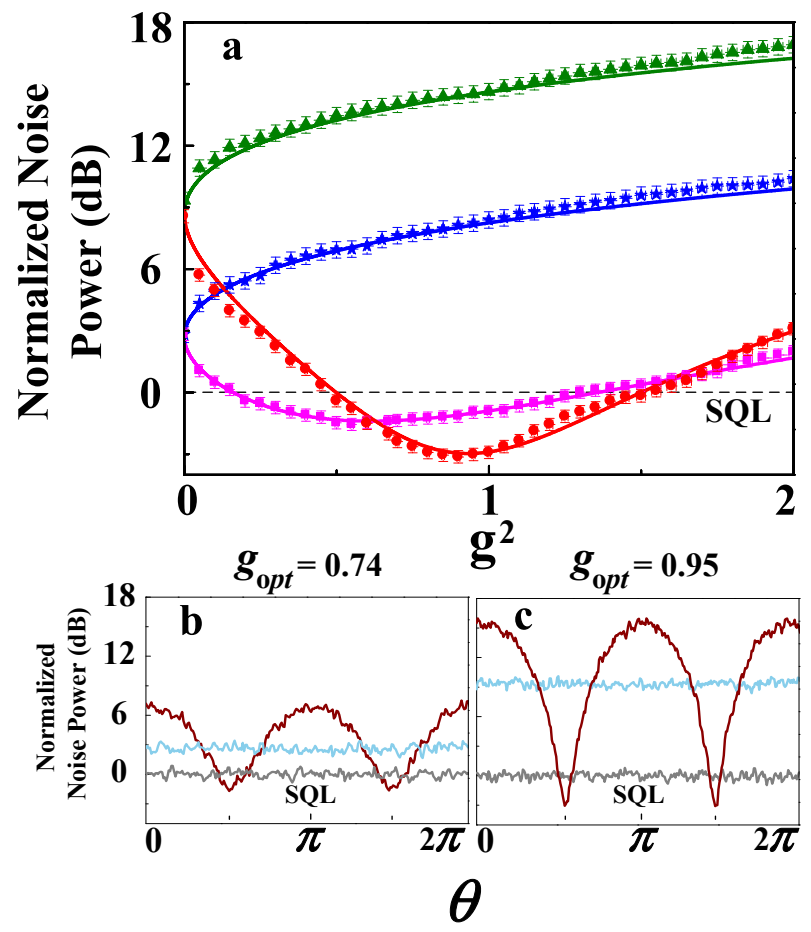

FIG. 2: The noise variance of the conditional quadrature phase amplitude. a, The noise variance of the conditional quadrature phase amplitude as the function of the factor $g^{2}$. The conditional quadrature phase (magenta squares) and amplitude (blue stars) are for the initial squeezing 3.9 $\mathrm{dB}$ (antisqueezing $5.24 \mathrm{~dB}$ ) and the extra noise $N_{e}=1.75$. The conditional quadrature phase (red circles) and amplitude (green triangles) are for the initial squeezing $5.9 \mathrm{~dB}$ (antisqueezing $11.6 \mathrm{~dB}$ ) and the extra noise $N_{e}=21.1$. All the solid curves are theoretical fitting according to different experimental parameters. $\mathbf{b}$, The noise variance of the conditional arbitrary quadrature components with the initial squeezing $3.9 \mathrm{~dB}$ (antisqueezing $5.24 \mathrm{~dB}$ ), the extra noise $N_{e}=1.75$ and the optimal factor $g_{\text {opt }}=0.74$. c, The noise variance of the conditional arbitrary quadrature components with the initial squeezing $5.9 \mathrm{~dB}$ (antisqueezing $11.6 \mathrm{~dB}$ ), the extra noise $N_{e}=21.1$ and the optimal factor $g_{\text {opt }}=0.95$. The blue (light gray) curves in (b) and (c) are the noise variance of the arbitrary quadrature components of the lower sideband field of the squeezing light. $\mathrm{RBW}=30 \mathrm{kHz}, \mathrm{VBW}=30 \mathrm{~Hz}$ and sweep time $=500 \mathrm{~ms}$.

$3.9 \mathrm{~dB}$ and $3.1 \mathrm{~dB}$ for that of $5.9 \mathrm{~dB}$. Quantum advantage resulting from the use of squeezed light is evaluated by comparing signal-to-noise ratios in this work. Because of additional degrees of freedom such as the optical gain $\mathrm{g}$, a fair comparison between quantum and classical light can be difficult. However, the quantum noise floors of both the classical and the quantum approaches are compared after independent factor over $\mathrm{g}$ in this works, because changing $g$ here does not change the signal level, as evidenced in Fig. 3. In this scheme, one can avoid the low frequency technical noise of the squeezed-light source by placing the signal and the detection in one of 


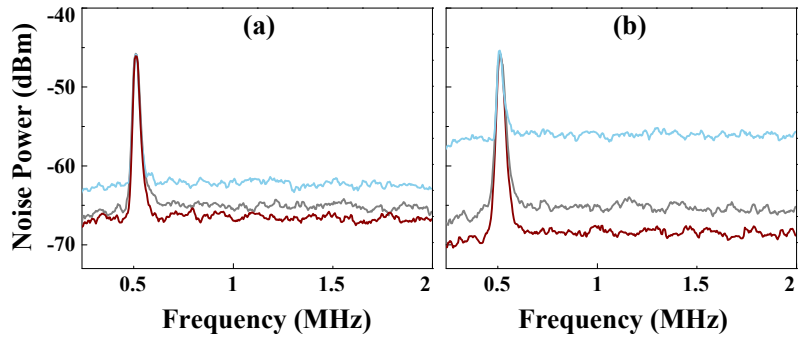

FIG. 3: The noise spectra of the low-frequency signal with $500 \mathrm{kHz}$ around the frequency $\omega_{0}$ by using broad squeezed light and BLO. a, The initial squeezing is 3.9 $\mathrm{dB}\left(e^{-2 r}=0.4\right)$ and the extra noise is $N_{e}=1.75$. bo, The initial squeezing is $5.9 \mathrm{~dB}\left(e^{-2 r}=0.26\right)$ and the extra noise is $N_{e}=21.1$. The black (gray) curves are the noise spectrum of the signal field with the input vacuum field and $g=0$. The blue (light gray) curves are the noise spectrum of the signal field with the input squeezed field and $g=0$. The red (dark gray) curves are the noise spectrum of the signal field with the input squeezed field and $g_{\text {opt }} . \mathrm{RBW}=10 \mathrm{kHz}, \mathrm{VBW}=30$ $\mathrm{Hz}$.

the squeezed sidebands. This is useful only if it is effectively the dominant source of noise. On the other hand, the low frequency noise introduced by homodyne detection can not avoid in the scheme. For example, amplitude noise will be rejected by the balanced detector, up to the common-mode rejection power. However, phase noise on will still creep in, particularly if the squeezing is strong.

In conclusion, we have demonstrated a scheme of using a broadband high-frequency squeezed vacuum to detect low-frequency signal beyond the standard quantum limit. By means of the EPR entanglement of upper and lower sideband of the broadband squeezed light, the conditional variance in the low frequency band can be obtained by BLO detection with subtracting the photocurrent of the upper sideband beam from that of the lower sideband beam. Thus this scheme does not need directly generate the squeezing in the low frequency band. In addition, the BLO detection directly measures the signal mapped on the sideband of the squeezed state, in this sense this scheme can to some extent avoid the DC technical noise in the traditional homodyne detection stemming from the light sources.

The authors would like to thank Yiqiu Ma for helpful discussion. This research is supported by the MOST (Grant No. 2016YFA0301602), NSFC (Grant No. 11234008, 11361161002, 11474188,11654002,6157127), Natural Science Foundation of Shanxi Province (Grant No. 2015011007), Research Project Supported by Shanxi Scholarship Council of China (Grant No. 2015-002).

${ }^{\dagger}$ Corresponding author email: jzhang74@sxu.edu.cn, jzhang74@yahoo.com.

APPENDIX:The conditional squeezing with he extra noise of the antisqueezed component

Usually, the broadband squeezed light generated by
OPO is not the minimum uncertainty state, in which the antisqueezed component has the extra noise. So the phase amplitudes of the output lower and upper sideband fields of OPO with the extra noise can be given by

$$
\begin{aligned}
& \hat{X}_{-}^{s}(\Omega)=\hat{X}_{-}^{0}(\Omega) \cosh r+\hat{X}_{+}^{0}(\Omega) \sinh r+\frac{N_{X}}{2}, \\
& \hat{Y}_{-}^{s}(\Omega)=\hat{Y}_{-}^{0}(\Omega) \cosh r-\hat{Y}_{+}^{0}(\Omega) \sinh r+\frac{N_{Y}}{2}, \\
& \hat{X}_{+}^{s}(\Omega)=\hat{X}_{+}^{0}(\Omega) \cosh r+\hat{X}_{-}^{0}(\Omega) \sinh r+\frac{N_{X}}{2}, \\
& \hat{Y}_{+}^{s}(\Omega)=\hat{Y}_{+}^{0}(\Omega) \cosh r-\hat{Y}_{-}^{0}(\Omega) \sinh r-\frac{N_{Y}}{2},
\end{aligned}
$$

here, $\left\langle\delta^{2} N_{X}\right\rangle=\left\langle\delta^{2} N_{Y}\right\rangle=N_{e}$. The difference and sum of amplitude phase quadratures of the output lower and upper sideband fields of OPO are obtained

$$
\begin{aligned}
\hat{X}_{-}^{s}(\Omega)-\hat{X}_{+}^{s}(\Omega) & =\left(\hat{X}_{-}^{0}(\Omega)-\hat{X}_{+}^{0}(\Omega)\right) e^{-r}, \\
\hat{Y}_{-}^{s}(\Omega)+\hat{Y}_{+}^{s}(\Omega) & =\left(\hat{Y}_{-}^{0}(\Omega)+\hat{Y}_{+}^{0}(\Omega)\right) e^{-r} \\
\hat{X}_{-}^{s}(\Omega)+\hat{X}_{+}^{s}(\Omega) & =\left(\hat{X}_{-}^{0}(\Omega)+\hat{X}_{+}^{0}(\Omega)\right) e^{+r}+N_{X}, \\
\hat{Y}_{-}^{s}(\Omega)-\hat{Y}_{+}^{s}(\Omega) & =\left(\hat{Y}_{-}^{0}(\Omega)-\hat{Y}_{+}^{0}(\Omega)\right) e^{+r}+N_{Y} \cdot(12)
\end{aligned}
$$

The variances of the output lower and upper sideband fields of OPO are expressed by

$$
\begin{aligned}
\left\langle\delta^{2} X_{-}^{s}(\Omega)\right\rangle & =\left\langle\delta^{2} X_{+}^{s}(\Omega)\right\rangle=\left\langle\delta^{2} Y_{-}^{s}(\Omega)\right\rangle=\left\langle\delta^{2} Y_{+}^{s}(\Omega)\right\rangle \\
& =\frac{e^{-2 r}+e^{2 r}}{2}+\frac{N_{e}}{4}
\end{aligned}
$$

and the correlated variances are given by

$$
\begin{aligned}
\left\langle\delta^{2}\left(\hat{X}_{-}^{s}(\Omega)-\hat{X}_{+}^{s}\right)(\Omega)\right\rangle & =\left\langle\delta^{2}\left(\hat{Y}_{-}^{s}(\Omega)+\hat{Y}_{+}^{s}(\Omega)\right)\right\rangle=2 e^{-2 r} \\
\left\langle\delta^{2}\left(\hat{X}_{-}^{s}(\Omega)+\hat{X}_{+}^{s}\right)(\Omega)\right\rangle & =\left\langle\delta^{2}\left(\hat{Y}_{-}^{s}(\Omega)-\hat{Y}_{+}^{s}(\Omega)\right)\right\rangle=2 e^{+2 r}+\left(\mathbb{N}_{\text {L }}\right)
\end{aligned}
$$

The variance of the conditional quadrature phase $\hat{Y}_{B}(\Omega)$ and amplitude $\hat{X}_{B}(\Omega)$ detected by the BLO with factor are expressed by

$$
\begin{aligned}
\left\langle\delta^{2} \hat{Y}_{B}(\Omega)\right\rangle & =\left\langle\delta^{2}\left(\hat{Y}_{-}^{s}+g \hat{Y}_{+}^{s}\right)\right\rangle \\
& =\frac{1}{2}\left(e^{2 r}+\frac{N_{e}}{2}\right)(1-g)^{2}+\frac{e^{-2 r}}{2}(1+g)^{2}, \\
\left\langle\delta^{2} \hat{X}_{B}(\Omega)\right\rangle & =\left\langle\delta^{2}\left(\hat{X}_{-}^{s}+g \hat{X}_{+}^{s}\right)\right\rangle \\
& =\frac{1}{2}\left(e^{2 r}+\frac{N_{e}}{2}\right)(1+g)^{2}+\frac{e^{-2 r}}{2}(1-g)^{2}(15)
\end{aligned}
$$

When we choose the optimized value of $g_{\text {opt }}=\left(e^{2 r}+\right.$ $\left.N_{e} / 2-e^{-2 r}\right) /\left(e^{2 r}+N_{e} / 2+e^{-2 r}\right)$, the minimum conditional variance of the output lower sideband field is obtained

$$
\left\langle\delta^{2} \hat{Y}_{B}^{o p t}(\Omega)\right\rangle=\frac{2 e^{-2 r}\left(e^{2 r}+N_{e} / 2\right)}{e^{2 r}+N_{e} / 2+e^{-2 r}} .
$$


At the same time, we may give the quadrature amplitude $\hat{Y}_{B}(\Omega)$ with the same condition is expressed by

$$
\begin{aligned}
\left\langle\delta^{2} \hat{X}_{B}(\Omega)\right\rangle & =\left\langle\delta^{2}\left(\hat{Y}_{-}^{s}+g \hat{Y}_{+}^{s}\right)\right\rangle \\
& =\frac{1}{2}\left(e^{2 r}+\frac{N_{e}}{2}\right)(1-g)^{2}+\frac{e^{-2 r}}{2}(1+g)(17)
\end{aligned}
$$

[1] B. P. Abbott, et al. (LIGO Scientific collaboration and the Virgo Collaboration), Phys. Rev. Lett. 116, 061102 (2016).

[2] B. P. Abbott, et al. (LIGO Scientific collaboration and the Virgo Collaboration), Phys. Rev. Lett. 116, 131102 (2016).

[3] B. P. Abbott, et al. (LIGO Scientific collaboration and the Virgo Collaboration), Phys. Rev. Lett. 116, 131103 (2016).

[4] C. M. Caves, Phys. Rev. D 23, 1693 (1981).

[5] M. Xiao, L.-A. Wu, H. J. Kimble, Phys. Rev. Lett. 59, 278 (1987).

[6] P. Grangier, R. E. Slusher, B. Yurke, A. LaPorta, Phys. Rev. Lett. 59, 2153 (1987).

[7] LIGO Scientific collaboration, Nature Phys. 7, 962 (2011).

[8] LIGO Scientific collaboration, Nature Photonics. 7, 613 (2013).

[9] H. J. Kimble, Y. Levin, A. B. Matsko, K. S. Thorne, S. P. Vyatchanin, Phys. Rev. D 65, 022002 (2001).

[10] S. Chelkowski, H. Vahlbruch, B. Hage, A. Franzen, N. Lastzka, K. Danzmann, R. Schnabel, Phys. Rev. A 71, 013806 (2005).

[11] J. Laurat, T. Coudreau, G. Keller, N. Treps, and C. Fabre, Phy. Rev. A 70, 042315 (2004).

[12] C. F. McCormick, A. M. Marino, V. Boyer, and P. D. Lett, Phy. Rev. A 78, 043816 (2008).

[13] T. Horrom, R. Singh, J. P. Dowling, and E. E. Mikhailov, Phy. Rev. A 86, 023803 (2012).

[14] N. Otterstrom, R. C. Pooser, and B. J. Lawrie, Opt. Lett. 39, 6533 (2014).

[15] R. C. Pooser, and B. J. Lawrie, ACS Photonics 3, 8 (2016).

[16] W. J. Fan, B. J. Lawrie, and R. C. Pooser, Phy. Rev. A 92, 053812 (2015).

[17] A. Huck, S. Smolka, P. Lodahl, A. S. S?rensen, A. Boltasseva, J. Janousek, and U. L. Andersen, Phy. Rev. Lett.
102, 246802 (2009).

[18] M. W. Holtfrerich, M. Dowran, R. Davidson, B. J. Lawrie, R. C. Pooser, and A. M. Marino, Optica 3, 985 (2016).

[19] R. C. Pooser, and B. Lawrie, Optica 2, 393 (2015).

[20] U. B. Hoff, G. I. Harris, L. S. Madsen, H. Kerdoncuff, M. Lassen, B. M. Nielsen, W. P. Bowen, and U. L. Andersen, Opt. Lett. 38, 1413 (2013).

[21] J. D. Teufel, T. Donner, M. A. Castellanos-Beltran, J. W. Harlow, and K. W. Lehnert, Nature Nanotechnology 4, 820 (2009).

[22] V. Boyer, A. M. Marino, and P. D. Lett, Phy. Rev. Lett. 100, 143601 (2008).

[23] B. J. Lawrie and R. C. Pooser, Opt. Express 21, 7549 (2013).

[24] M. I. Kolobov, and C. Fabre, Phy. Rev. Lett. 85, 3789 (2000).

[25] J. Zhang, Phys. Rev. A 67, 054302 (2003).

[26] E. H. Huntington, G. N. Milford, C. Robilliard, T. C. Ralph, O. Glökl, U. L. Andersen, S. Lorenz, and G. Leuchs, Phys. Rev. A 71, 041802 (2005).

[27] B. Hage, A. Samblowski, and R. Schnabel, Phys. Rev. A 81, 062301 (2010).

[28] Y. Ma, H. Miao, B. H. Pang, M. Evans, C. Zhao, J. Harms, R. Schnabel, Y. Chen, arXiv:1612.06934

[29] A. M. Marino, C. R. Stroud, V. Wang, R. S. Bennink, and R. W. Boyd, J. Opt. Soc. Am. B 24, 335 (2007).

[30] H. Fan, D. He, and S. Feng, arXiv:1410.8602

[31] W. Li, X. Yu, Z. Meng, Y. Jin and J. Zhang, Sci. China-Phys. Mech. Astron. 58, 104201 (2015); arXiv:1508.04974 (2015).

[32] J. -B. Béguin, E. M. Bookjans, S. L. Christensen, H. L. Sørensen, J. H. Müller, E. S. Polzik, and J. Appel, Phy. Rev. Lett. 113, 263603 (2014).

[33] W. Li, X. D. Yu, and J. Zhang, Opt. Lett. 40, 5299 (2015).

[34] W. Li, Y. Jin, and X. Yu, Sci. China-Phys. Mech. Astron. 60, 050321 (2017).

[35] M. Pysher, Y. Miwa, R. Shahrokhshahi, R. Bloomer, and O. Pfister, Phy. Rev. Lett. 107, 030505 (2011).

[36] O. Pinel, P. Jian, R. M. de Araújo, J. Feng, B. Chalopin, C. Fabre, and N. Treps, Phy. Rev. Lett. 108, 083601 (2012).

[37] M. D. Reid, Phy. Rev. A 40, 913 (1989).

[38] A. M. Marino, R. C. Pooser, V. Boyer, P. D. Lett, Nature 457, 859 (2009). 